1 Hacettepe Journal of Mathematics and Statistics

$\bigcap$ Volume 43 (6) (2014), 891-898

\title{
On some variants of compactness
}

\author{
S. Bayhan * and I. L. Reilly ${ }^{\dagger}$
}

\begin{abstract}
Three weak variants of compactness were introduced and studied by Kohli and Singh [ Acta Math. Hungar. 106 (2005), 317-329 ]. These three properties are reconsidered from the change of topology perspective. In particular, it is shown that each of these properties is equivalent to compactness with respect to another topology on the underlying set. Some consequences of this situation are investigated.
\end{abstract}

2000 AMS Classification: 54D10, 54D20, 54D30, 54C08, 54C10.

Keywords: compactness, change of topology, $d$-compact, $d^{*}$-compact, $D_{\delta^{-}}$ compact, continuous function, $D$-continuous function, $D^{*}$-continuous function, $D_{\delta}$-continuous function, $D$-supercontinuous function, $D$-irresolute function

Received 12:07:2013 : Accepted $08: 11: 2013$ Doi : 10.15672/HJMS.2014437532

\section{Introduction}

The notion of compactness is one of the most significant topological properties, and its importance reaches well beyond topology to several other branches of mathematics. Weaker variants of compactness have been considered in the topological literature for at least nine decades. For example, Hausdorff almost compact spaces (now known as Hclosed spaces) were introduced by Alexandroff and Urysohn [1], and have subsequently been investigated by many researchers. The book [12] is a comprehensive source of references. Almost compactness is considered in the book [3]. Frolik [5] introduced quasicompact spaces. One reason for their significance is that functionally Hausdorff quasicompact spaces are the natural setting for the Stone-Weierstrass theorem, see Stephenson [17].

In 2005 Kohli and Singh [10] introduced three weak variants of compactness which lie between compactness and quasicompactness. They studied the basic properties of the

\footnotetext{
*Department of Mathematics, Mehmet Akif Ersoy University, 15030, Istiklal Campus, Burdur, Turkey

Email: bayhan@mehmetakif.edu.tr

†Department of Mathematics,University of Auckland,P. B. 92019, Auckland, New Zealand Email:i.reilly@auckland.ac.nz

The authors gratefully acknowledge financial support of this research by the Scientific and Technological Research Council of Turkey (TUBITAK).
} 
classes of spaces defined by these three weak variants of compactness, which have been named $d$-compactness, $d^{*}$-compactness and $D_{\delta}$-compactness.

In this paper, we reconsider these three notions from the perspective of change of topology. In each case, we observe that there is an appropriate change of topology which reveals that the new concept is equivalent to the classical notion of compactness. The last two results of Kohli and Singh [10, Theorems 5.17 and 5.18] make this observation almost as a post script. They do not use it anywhere in their paper [10]. In our view, this is the fundamental defining characteristic of these three notions of weak compactness. For us, it is the starting point of the discussion. Each of these three new notions is compactness with respect to another topology on the underlying set. This observation provides the natural setting for the subsequent discussion of each of these three notions.

We are able to exploit this observation to produce more elegant alternative proofs of some of the results of Kohli and Singh [10], and to suggest other results.

Our notation and terminology are standard, see for example Dugundji [4]. In particular, we do not assume any separation properties for the spaces we consider, unless explicitly stated. We denote the interior of a subset $B$ of the topological space $(X, \tau)$ by

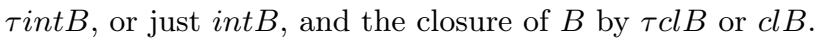

\section{Preliminaries and definitions}

In a topological space $(X, \tau)$ a set $B$ is defined to be regular open if $B=\operatorname{\tau int}(\tau c l B)$. Since the intersection of two regular open sets is regular open, the collection of all $\tau$ regular open sets forms the base for a topology $\tau_{s}$ on $X$, smaller than $\tau$, called the semi-regularization of $(X, \tau)$. Note that $(X, \tau)$ is semi-regular if and only if $\tau=\tau_{s}$.

In 1968 Veličko [18] made the following definition.

Let $(X, \tau)$ be a topological space and let $A \subset X$. A point $x \in X$ is called a $\theta$-limit point of $A \subset X$ if every closed neighbourhood of $X$ intersects $A$. Let $\theta c l A$ denote the set of all $\theta$-limit points of $A$. The set $A$ is $\theta$-closed if $A=\theta \operatorname{cl} A$. The complement of a $\theta$-closed set is called a $\theta$-open set. The collection of all $\theta$-open sets in $(X, \tau)$ forms a topology on $X$, denoted by $\tau_{\theta}$.

A subset $B$ of $(X, \tau)$ is called a zero-set if there is a continuous real-valued function $f$ defined on $X$ such that $B=\{x \in X: f(x)=0\}$. The complement of a zero-set is called a co-zero set. The collection of all co-zero sets of $(X, \tau)$ is the base for a topology $\tau_{z}$ on $X$, and $\tau_{z} \subset \tau$. Moreover, $(X, \tau)$ is completely regular if and only if $\tau_{z}=\tau$.

2.1. Definition A space $(X, \tau)$ is said to be

(1) almost compact [3] if every open cover of $X$ has a finite subcollection the closures of whose members cover $X$;

(2) quasicompact [5] if every cover of $X$ by co-zero sets has a finite subcover;

(3) nearly compact [14] if every open cover of $X$ admits a finite subcollection the interiors of the closures of whose members cover $X$;

(4) $\theta$-compact [13, Definition 3.19] if every cover of $X$ by $\theta$-open sets has a finite subcover.

The following result was proved by Carnahan [2, Theorem 4.1].

2.2. Theorem $(X, \tau)$ is nearly compact if and only if $\left(X, \tau_{s}\right)$ is compact. 
Definition 2.1 (2) immediately implies that

\subsection{Theorem}

(1) $(X, \tau)$ is quasicompact if and only if $\left(X, \tau_{z}\right)$ is compact.

(2) $(X, \tau)$ is $\theta$-compact if and only if $\left(X, \tau_{\theta}\right)$ is compact [13, Remark 4.27].

Following Heldermann [6] we have two definitions.

2.4. Definition A collection $\beta$ of subsets of a space $(X, \tau)$ is called an open complementary system if $\beta$ consists of open sets such that for every $B \in \beta$, there exist $B_{1}, B_{2}, \ldots \in \beta$ with $B=\cup\left\{X / B_{i}: i \in \mathbb{N}\right\}$.

2.5. Definition A subset $U$ of a space $(X, \tau)$ is called a strongly open $F_{\sigma}$-set if there exists a countable open complementary system $\beta(U)$ with $U \in \beta(U)$. The complement of a strongly open $F_{\sigma}$-set is called a strongly closed $G_{\delta}$-set.

Mack [11] made the next definition in 1970.

2.6. Definition A subset $H$ of a space $(X, \tau)$ is called a regular $G_{\delta}$-set if $H$ is the intersection of a sequence of closed sets whose interiors contain $H$, i.e., if $H=\bigcap_{n=1}^{\infty} F_{n}=$ $\bigcap_{n=1}^{\infty} i n t F_{n}$, where each $F_{n}$ is a closed subset of $X$. The complement of a regular $G_{\delta}$-set is called a regular $F_{\sigma}$-set.

Kohli and Singh [10] introduced three weak variants of compactness which we now consider from the perspective of change of topology.

2.7. Definition A space $(X, \tau)$ is said to be $d$-compact $\left(d^{*}\right.$-compact, $D_{\delta}$-compact) if every cover of $X$ by open $F_{\sigma}$-sets (strongly open $F_{\sigma}$-sets, regular $F_{\sigma}$-sets) has a finite subcover.

\section{Three topologies}

Let $(X, \tau)$ be a topological space, and denote by $\beta$ the collection of all open $F_{\sigma}$-subsets of $(X, \tau)$. Now the intersection of two open $F_{\sigma}$-subsets is an open $F_{\sigma}$-subset. Therefore the collection $\beta$ is a base for a topology on $X$, which we denote by $\tau_{d}$. This topology $\tau_{d}$ is called the $D$-regularization of $\tau$ by Kohli and Singh [10].

Similarly, if we replace " open $F_{\sigma}$-subsets " in the paragraph immediately above by " strongly open $F_{\sigma}$-subsets ", we obtain a second topology on $X$, denoted by $\tau^{*}$, and called the $D$-complete regularization of $\tau$ in [10].

Yet again, if we replace " open $F_{\sigma}$-subsets " by " regular $F_{\sigma}$-subsets " we obtain a third topology on $X$, denoted by $\tau^{\#}$, and called the $D_{\delta}$-complete regularization of $\tau$ by Kohli and Singh [10].

There is an alternative way of defining these three topologies given by the next definition.

3.1. Definition A set $G$ in a topological space $(X, \tau)$ is said to be $\tau_{d}$-open [8] ( $\tau^{*}$-open, $\tau^{\#}$-open) if for each $x \in G$, there exists an open $F_{\sigma^{-}}$set (strongly open $F_{\sigma^{-}}$ set, regular $F_{\sigma}$-set) $H$ such that $x \in H \subset G$. The complement of a $\tau_{d}$-open $\left(\tau^{*}\right.$-open, $\tau^{\#}$-open) set will be referred to as a $\tau_{d}$-closed $\left(\tau^{*}\right.$-closed, $\tau^{\#}$-closed) set. 
We note that the members of these topologies are denoted by $d$-open, $d^{*}$-open and $d^{\#}$-open sets in [8], [15] and [9] respectively.

\section{Change of topology}

The fundamental defining characteristic of each of the three weak variants of compactness that we are considering is given by the following result, which is proved immediately from the definitions. Kohli and Singh [10, Theorems 5.17 and 5.18] have made this observation.

4.1. Theorem Let $(X, \tau)$ be a topological space. Then

(1) $(X, \tau)$ is $d$-compact if and only if $\left(X, \tau_{d}\right)$ is compact,

(2) $(X, \tau)$ is $d^{*}$-compact if and only if $\left(X, \tau^{*}\right)$ is compact,

(3) $(X, \tau)$ is $D_{\delta}$-compact if and only if $\left(X, \tau^{\#}\right)$ is compact.

Kohli and Singh [10] have provided an impressive list of Examples (2.8 to 2.13) to show all the weak variants shown in their diagram of relationships are distinct. We reproduce their diagram of relationships here as Figure 1.

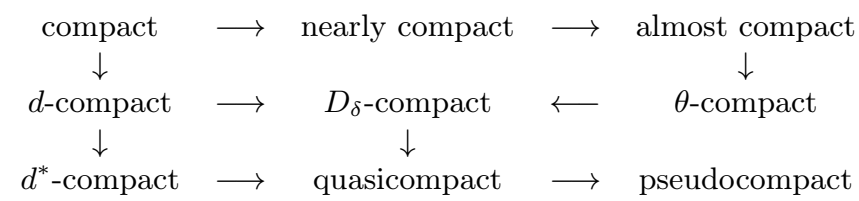

Figure 1.

We take this diagram to mean that one can find a topological space $(X, \tau)$ having one of these properties but not one of the stronger properties. For this interpretation of Figure 1 one must regard the topology on $X$ as fixed. Theorems 2.2, 2.3 and 4.1 indicate that six of the concepts in Figure 1 are each separately equivalent to compactness provided an appropriate change of the topology on the underlying set $X$ is made in each case. It seems that the two exceptions are almost compactness and pseudocompactness.

Claims of the kind that " $d$-compactness is independent of compactness" are confusing. In fact, $d$-compactness is a disguised form of compactness. It is compactness with respect to another topology on the underlying set. So $d$-compactness is not a new concept. It is equivalent to the classical notion of compactness, only with respect to a different topology (than the original topology) on the underlying set. The same comments apply to the notions of $d^{*}$-compactness and $D_{\delta}$-compactness.

\section{Some Basic Properties}

The following definition is due to Kohli and Singh [10, Definition 3.2]

5.1. Definition A topological space $(X, \tau)$ said to be $D$-Hausdorff ( $D^{*}$-Hausdorff, $D_{\delta}$-Hausdorff) if each pair of distinct points is contained in disjoint open $F_{\sigma}$-sets (strongly open $F_{\sigma}$-sets, regular $F_{\sigma}$-sets).

The proof of the next result is immediate from Definitions 5.1 and 3.1. 
5.2. Proposition Let $(X, \tau)$ be a topological space. Then

(1) $(X, \tau)$ is $D$-Hausdorff if and only if $\left(X, \tau_{d}\right)$ is Hausdorff.

(2) $(X, \tau)$ is $D^{*}$-Hausdorff if and only if $\left(X, \tau^{*}\right)$ is Hausdorff.

(3) $(X, \tau)$ is $D_{\delta}$-Hausdorff if and only if $\left(X, \tau^{\#}\right)$ is Hausdorff.

One of the well-known standard results of a first course in topology, for example see Dugundji [4, page 224 Theorem 1.4 (2)], is the following proposition.

5.3. Proposition If $(X, \tau)$ is a Hausdorff space and $A$ is a compact subset $(X, \tau)$, then $A$ is closed in $(X, \tau)$.

Applying Theorem 4.1 and Propositions 5.2 and 5.3 we obtain the next result.

5.4. Proposition If $A$ is a $d$-compact subset of the $D$-Hausdorff space $(X, \tau)$, then $A$ is $d$-closed in $X$.

Proof. Observe that $A$ is a compact subset of the Hausdorff space $\left(X, \tau_{d}\right)$, so that Proposition 5.3 implies that $A$ is closed in $\left(X, \tau_{d}\right)$, or that $A$ is $d$-closed in $(X, \tau)$.

Exactly parallel results can be obtained by analogous proofs for a $d^{*}$-compact $\left(D_{\delta^{-}}\right.$ compact) subset of a $D^{*}$-Hausdorff $\left(D_{\delta}\right.$-Hausdorff) space. These three results have been proved from first principles by Kohli and Singh [10, Theorem 3.3].

Another standard result concerning compact spaces is that a closed subset of a compact space is compact, for example see Dugundji [4, page 224 Theorem 1.4 (3)]. From this result we obtain the following result which generalizes Theorem 3.10 of [10].

5.5. Proposition Let $(X, \tau)$ be a $d$-compact topological space, and $A$ be $\tau_{d}$-closed in $X$. Then $A$ is $d$-compact.

Again, we can provide exactly parallel results for the other two variants of compactness.

5.6. Proposition Let $(X, \tau)$ be a $d^{*}$-compact ( $D_{\delta}$-compact) topological space, and $A$ be $\tau^{*}$-closed $\left(\tau^{\#}\right.$-closed) in $X$. Then $A$ is $d^{*}$-compact ( $D_{\delta}$-compact).

It is well-known that compactness can be characterized in terms of the finite intersection property and adherence properties of filters and filterbases, see Dugundji [4, page 223 Theorem 1.3] for example. Kohli and Singh [10, Theorem 4.4] provide a version of these characterizations for $d$-compactness, $d^{*}$-compactness and $D_{\delta}$-compactness. We note that their definitions of $d$-adherence and $d$-convergence of a filterbase $T$ [10, Definitions 4.2 and 4.3] are equivalent to adherence and convergence of $T$ with respect to the topology $\tau_{d}$. A change of topology approach to this topic is an alternative to the discussion presented in Section 4 of [10].

In order to consider mapping properties we must define appropriate classes of functions between topological spaces.

5.7. Definition A function $f:(X, \tau) \longrightarrow(Y, \sigma)$ is defined to be $D$-continuous [7] ( $D^{*}$-continuous [16], $D_{\delta}$-continuous) if for each point $x \in X$ and each open $F_{\sigma}$-set (strongly open $F_{\sigma}$-set, regular $F_{\sigma}$-set) $V$ containing $f(x)$ there is an open subset $U$ of $X$ such that $x \in U$ and $f(U) \subset V$. 
5.8. Definition A function $f:(X, \tau) \longrightarrow(Y, \sigma)$ is defined to be $D$-supercontinuous [8] ( $D^{*}$-supercontinuous [15], $D_{\delta}$-supercontinuous [9]) if for each point $x \in X$ and each open $V$ of $X$ containing $f(x)$ there is an open $F_{\sigma}$-set (strongly open $F_{\sigma}$-set, regular $F_{\sigma}$-set) $U$ in $X$ such that $x \in U$ and $f(U) \subset V$.

5.9. Definition A function $f:(X, \tau) \longrightarrow(Y, \sigma)$ is defined to be $D$-irresolute $\left(D^{*}\right.$ irresolute, $D_{\delta}$-irresolute) if for each point $x \in X$ and each open $F_{\sigma}$-set (strongly open $F_{\sigma}$-set, regular $F_{\sigma}$-set) $V$ containing $f(x)$ there is an open $F_{\sigma}$-set (strongly open $F_{\sigma}$-set, regular $F_{\sigma}$-set) $U$ in $X$ such that $x \in U$ and $f(U) \subset V$.

The following results are immediate from the preceding definitions and the discussion in Section 3.

5.10. Proposition Let $f:(X, \tau) \longrightarrow(Y, \sigma)$ be a function between topological spaces. Then

(1) $f:(X, \tau) \longrightarrow(Y, \sigma)$ is $D$-continuous if and only if $f:(X, \tau) \longrightarrow\left(Y, \sigma_{d}\right)$ is continuous.

(2) $f:(X, \tau) \longrightarrow(Y, \sigma)$ is $D^{*}$-continuous if and only if $f:(X, \tau) \longrightarrow\left(Y, \sigma^{*}\right)$ is continuous.

(3) $f:(X, \tau) \longrightarrow(Y, \sigma)$ is $D_{\delta}$-continuous if and only if $f:(X, \tau) \longrightarrow\left(Y, \sigma^{\#}\right)$ is continuous.

(4) $f:(X, \tau) \longrightarrow(Y, \sigma)$ is $D$-supercontinuous if and only if $f:\left(X, \tau_{d}\right) \longrightarrow(Y, \sigma)$ is continuous.

(5) $f:(X, \tau) \longrightarrow(Y, \sigma)$ is $D^{*}$-supercontinuous if and only if $f:\left(X, \tau^{*}\right) \longrightarrow(Y, \sigma)$ is continuous.

(6) $f:(X, \tau) \longrightarrow(Y, \sigma)$ is $D_{\delta}$-supercontinuous if and only if $f:\left(X, \tau^{\#}\right) \longrightarrow(Y, \sigma)$ is continuous.

(7) $f:(X, \tau) \longrightarrow(Y, \sigma)$ is $D$-irresolute if and only if $f:\left(X, \tau_{d}\right) \longrightarrow\left(Y, \sigma_{d}\right)$ is continuous.

(8) $f:(X, \tau) \longrightarrow(Y, \sigma)$ is $D^{*}$-irresolute if and only if $f:\left(X, \tau^{*}\right) \longrightarrow\left(Y, \sigma^{*}\right)$ is continuous.

(9) $f:(X, \tau) \longrightarrow(Y, \sigma)$ is $D_{\delta}$-irresolute if and only if $f:\left(X, \tau^{\#}\right) \longrightarrow\left(Y, \sigma^{\#}\right)$ is continuous.

The standard result that compactness is preserved by continuous functions, Proposition 5.10 and Theorem 4.1 can be used to prove the next set of results.

5.11. Proposition Let $f:(X, \tau) \longrightarrow(Y, \sigma)$ be a surjection.

(A) If $f$ is $D$-continuous ( $D^{*}$-continuous, $D_{\delta}$-continuous) and $(X, \tau)$ is compact then $(Y, \sigma)$ is $d$-compact ( $d^{*}$-compact, $D_{\delta}$-compact).

(B) If $f$ is $D$-supercontinuous ( $D^{*}$-supercontinuous, $D_{\delta}$-supercontinuous) and $(X, \tau)$ is $d$-compact $\left(d^{*}\right.$-compact, $D_{\delta}$-compact) then $(Y, \sigma)$ is compact.

(C) If $f$ is $D$-irresolute $\left(D^{*}\right.$-irresolute, $D_{\delta}$-irresolute) and $(X, \tau)$ is $d$-compact $\left(d^{*}\right.$ compact, $D_{\delta}$-compact) then $(Y, \sigma)$ is $d$-compact ( $d^{*}$-compact, $D_{\delta}$-compact). 
Proof. We prove one case of each part only. The other cases have exactly similar proofs.

(A) For $D$-continuity: Now $f:(X, \tau) \longrightarrow\left(Y, \sigma_{d}\right)$ is a continuous surjection and $(X, \tau)$ is compact, so that $\left(Y, \sigma_{d}\right)$ is compact. Thus $(Y, \sigma)$ is $d$-compact.

(B) For $D^{*}$-supercontinuity: Now $f:\left(X, \tau^{*}\right) \longrightarrow(Y, \sigma)$ is a continuous surjection and $\left(X, \tau^{*}\right)$ is compact. Hence $(Y, \sigma)$ is compact.

(C) For $D_{\delta}$-irresoluteness: Now $f:\left(X, \tau^{\#}\right) \longrightarrow\left(Y, \sigma^{\#}\right)$ is a continuous surjection and $\left(X, \tau^{\#}\right)$ is compact. Therefore $\left(Y, \sigma^{\#}\right)$ is compact, so that $(Y, \sigma)$ is $D_{\delta}$-compact.

Note that $5.11(\mathrm{~A})$ is Theorems 5.2 and 5.3 of Kohli and Singh [10], while $5.11(\mathrm{~B})$ is Theorems 5.5 and 5.6 of [10]. The proofs provided by Kohli and Singh [10] are from first principles, and quite different in character to the proofs given above.

The change of topology approach can be used to suggest new results. To illustrate, we provide two such results.

5.12. Proposition Let $f, g:(X, \tau) \longrightarrow(Y, \sigma)$ be $D$-irresolute, and $(Y, \sigma)$ be $D$ Hausdorff. Then $E$, the equalizer of $f$ and $g$, given by $E=\{x \in X: f(x)=g(x)\}$ is $d$-closed in $(X, \tau)$.

Proof. We have that $f, g:\left(X, \tau_{d}\right) \longrightarrow\left(Y, \sigma_{d}\right)$ are continuous, and that $\left(Y, \sigma_{d}\right)$ is Hausdorff. Thus by a standard result, e.g. Dugundji [4, page 140,1.5(1)], we have that $E$ is closed in $\left(X, \tau_{d}\right)$, so that $E$ is $d$-closed in $(X, \tau)$.

5.13. Proposition If $f:(X, \tau) \longrightarrow(Y, \sigma)$ is $D_{\delta}$-irresolute and $(Y, \sigma)$ is $D_{\delta}$-Hausdorff, then $G(f)$, the graph of $f$, is closed in $\left(X \times Y, \tau^{\#} \times \sigma^{\#}\right)$.

Proof. Note that $f:\left(X, \tau^{\#}\right) \longrightarrow\left(Y, \sigma^{\#}\right)$ is continuous, and that $\left(Y, \sigma^{\#}\right)$ is Hausdorff. Then a standard result for continuous functions, e.g. Dugundji [4, page 140, 1.5(3)], implies that $G(f)$ is closed in $\left(X \times Y, \tau^{\#} \times \sigma^{\#}\right)$.

\section{References}

[1] P. S. Alexandroff and P. Urysohn, Zur theorie der topologischen raume, Math. Ann. 92 (1924), 258-266.

[2] D. Carnahan, Locally nearly compact spaces, Boll. U. M. I. 4 (1972), 146-153.

[3] A. Császár, General Topology, Adam Hilger Ltd. Bristol, 1978.

[4] J. Dugundji, Topology, Allyn and Bacon, Boston, Mass. 1966.

[5] Z. Frolik, Generalizations of compact and Lindelöf spaces, Czech. Math. J. 13 (1959), 172-217.

[6] N. C. Helderman, Developability and some new regularity axioms, Can. J. Math., 33 (1981), 641-663.

[7] J. K. Kohli, $D$-continuous functions, $D$-regular spaces and $D$-Hausdorff spaces, Bull. Cal. Math. Soc., 84 (1992), 39-46. 
[8] J. K. Kohli and D. Singh, D-supercontinuous functions, Indian J. Pure Appl. Math., 32 (2001), 227-235.

[9] J. K. Kohli and D. Singh, $D_{\delta}$-supercontinuous functions, Indian J. Pure Appl. Math., 34 (2003), 1089-1100.

[10] J. K. Kohli and D. Singh, Between compactness and quasicompactness, Acta Math. Hungar., 106 (2005), 317-329.

[11] J. Mack, Countable paracompactness and weak normality properties, Trans. Amer. Math. Soc., 148 (1970), 265-272.

[12] J. R. Porter and R. G. Woods, Extensions and Absolutes of Hausdorff spaces, Springer-Verlag, New York, 1978.

[13] M. Saleh, On $\theta$-closed sets and some forms of continuity, Archivum Mathematicum (BRNO), 40 (2004), 383-393.

[14] M. K. Singal and A. Mather, On nearly-compact spaces, Boll. U. M. I., 2 (1969), 702-710.

[15] D. Singh, $D^{*}$-supercontinuous functions, Bull. Cal. Math. Soc., 94 (2002), 67-76.

[16] D. Singh, $D^{*}$-continuous functions, Bull. Cal. Math. Soc., 91 (1999), 385-390.

[17] R. M. Stephenson, Jr., Spaces for which the Stone-Weierstrass theorem holds, Trans. Amer. Math. Soc., 133 (1968), 537-546.

[18] N. Veličko, H-closed topological spaces, Amer. Math. Soc. Transl., 78 (2) (1968), 103-118. 